\title{
A Percolation Based Upscaling Technique for Viscous Force Dominated Waterflooding in Uncorrelated Heterogeneous Reservoirs
}

\author{
* Bedriḳovetsky P., "* Bruining J. \\ * Moscow Gubkin Oil \& Gas Academy, Russia \\ ** Delft Univ. of Technology, The Netherlands
}

Copyright 1995. Steering Committee of the European IOR - Symposium.

This paper was presented at the Eth. European IOR - Symposium in Vienna, Austria, May 16 - 17, 1996

This paper was elected for presentation by the Steering Committee, following roview of information conteined in an abstract

eubmitted by the author(s). The paper, as presented has not been reviewed by the Steering Committee.

\section{Abstract}

We present a percolation model of relative permeability for the case where viscous forces are dominant. In this respect the model differs from conventional percolation models of scale up of phase permeability where capillary forces control. The essential concepts are based on a one-phase analog for a heterogeneous reservoir that contains impermeable parts (e.g. shale). For two phases the percolation model uses only the permeability distribution and the end point permeabilities as input. The resulting relative permeabilities are compared with pseudo curves obtained for layered systems and pseudo curves for capillary dominated systems.

\section{Introduction}

Fluid displacement in heterogeneous reservoirs can be characterised by capillary, viscous and gravitational forces. [Yortsos (1991), Yortsos (1992), Kanevskaya (1988), Bedrikovetsky (1993)]. For water displacing oil from a water wet reservoir the capillary forces prefer the water in the low permeability regions whereas the viscous forces drive the water in the high permeable parts. Due to gravity the water will be in the bottom part of the reservoir [Dietz (1950)]. In a textbook example [Dake (1978)] of layer cake reservoirs capillary pressure equilibrium (vertical equilibrium) is assumed in the cross-dip direction and thus water prefers the low permeability layer. The condition is that the height of the layer $\mathrm{H}$ is much smaller than its length $\mathrm{L}$. This gives the fluids sufficient time to redistribute (over the height $\mathrm{H}$ ) according to capillary and gravitational pressure equilibrium before the water has swept the reservoir over its full length. The extreme opposite approach has been adopted by Hearn (1971) in his celebrated article. He assumes that viscous forces are dominant and that the displacing fluid fills the layers in order of decreasing permeability. Also in a capillary bundle model of a porous medium it appears that the displacing phase, e.g. water, first enters the tubes with the larger radii even in water wet reservoirs. Only under conditions of controlled (slowed) imbibition are capillary forces dominant. Hence the picture that emerges is that viscous forces dominate the flow at the displacement front and capillary and gravitational forces redistribute the fluids 


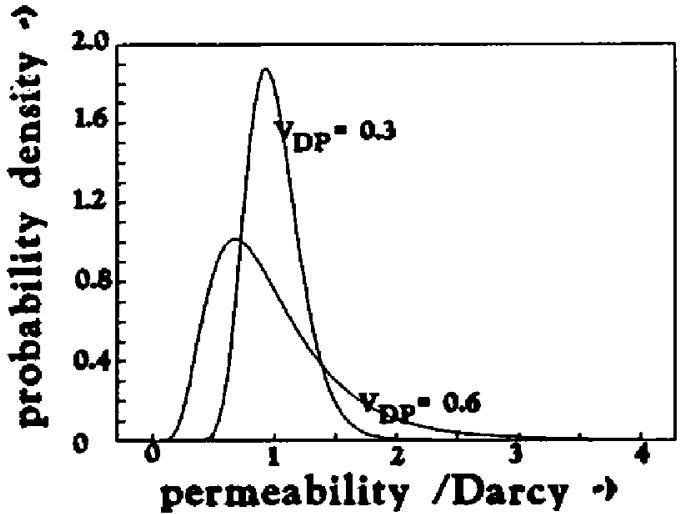

Figure 1: Log-normal permeability distribution functions $\psi(k)$ for a relatively homogeneous reservoir $\left(V_{D P}=0.3\right)$ and for a moderately heterogeneous reservoir $\left(V_{D P}=0.6\right.$.)

further upstream.

As Hearn (1971) and Dake (1978) represent the partially correlated case, (i.e. the permeability in the layer direction is fully correlated) our interest focusses on water flooding in a fully uncorrelated heterogeneous permeability field. Uncorrelated means that permeabilities in different parts of the reservoir are independent random functions with the same probability distribution function (see Figure 1).

We apply site percolation theory for the description of transport in a regular cubic lattice with random conductivity of the sites. Site percolation deals with occupation of lattice sites as opposed to bond percolation, which deals with open or closed bonds between the lattice sites. The approach is based on the generalised De Gennes-Sholovsky percolation model for a lattice with varying conductivity [Kadet and Selyakov (1987), Selyakov and Kadet (1995), Selyakov (1986)].

A percolation model for water-flooding in a reservoir with a stochastic heterogeneity was developed for the capillary dominant case by Yortsos (1991b, 1993) (see also Heiba, Sahimi et. al (1982), Heiba, Scriven, Davis (1984)). Here we develop a model for the viscous dominated case.

In section 2 we present the percolation model for one-phass flow. This model carries all the essential ideas. In section 3 we present the model for two-phase flow. In section 4 we compare the phase permeability and fractional flow functions for the correlated (layer) models with the functions for the completely uncorrelated percolation models. The equations for the capillary dominated case are stated in the appentdix: Introduction of the pseudo relative permeability results in a first order hyperbolic differential equation in terms of the average water saturation i.e. the Buckley Leverett equation. This equation describes a water flood in large scale approximation [Bedrikovetsky (1993), Coats (1971), Hearn (1971)]. The theory can also be extended for upscaling of the Leverett $J$ function, based on the self similar solution for the capillary imbibition problem [Barenblatt et al (1990), Ryzhik (1960), Bedrikovetsky, Bruining (1995)] but this is considered outside the scope of the present article. These aspects are discussed in section 5 .

\section{Upscaling for one-phase flow}

We consider a 3-D heterogeneous uncorrelated permeability field. We consider that a fraction $1-\chi$ is occupied by randomly distributed impermeable parts e.g. shales. The remaining fraction $\chi$ is occupied by permeable formation e.g. sand. The model assumes isotropic conditions and hence leads to isotropic average permeabilities (see, however, Haldorsen and Lake (1984)).

The permeability of the sand is distributed randomly with a probabilistic density function $\psi(k)$. Typical (log-normal see Jensen and Lake (1988)) distribution functions are shown in Figure 1. The curves are typical for a more or less homogeneous reservoir (Dykstra and Parsons' (1950) coefficient $V_{D P}=0.3$ ) and a slightly heterogeneous reservoir $\left(V_{D P}=0.6\right)$. The coefficient $V_{D P}=1-\exp (-s)$, where $s$ is the standard deviation of the log-normal permeability field. For convenience we have taken the average permeability at one (Darcy). These curves are used in all computations below.

If the fraction of sand $(\chi)$ drops below the percolation threshold $p_{c}$, sand will only form finite clusters and the effective permeability will be zero. The percolation threshold value $p_{c}$ is determined by the filling of the lattice $f$ and the dimension $D$ of the problem. For a 3-D lattice $\dot{p}_{c}=\frac{0.16}{f}$ and for the filling we assume that 
each site is occupied by a sphere with a radius of half the bond distance between sites [Efros (1982), Staufer (1985)].

$$
p_{c}=\frac{0.16}{0.52}=0.317
$$

Following the Sholovsky-De Gennes model we infer that the correlation radius is given by

$$
R(\chi)=\left(\frac{\chi-p_{c}}{1-p_{c}}\right)^{-\nu} \quad \chi>p_{c}
$$

where $\nu=0.9$ for 3-D problems. $R(\chi)$ is some average distance (correlation length) of two sites belonging to the same infinite cluster. The probability density that two sites belong to the same cluster decreases with the distance between the sites and tends to zero. Equation 2 is consistent with the correct asymptotic behaviour near the percolation threshold and the correct behaviour for no shale i.e. $\chi=1$ for which the cluster coincides with the original grid and the distance between neighbouring infinite clusters is one. It also ensures that $R$ decreases monotonically with $\chi$. In the same way we infer the expression for the length of the path between two sites belonging to the same infinite cluster.

$$
L(\chi)=\left(\frac{\chi-p_{c}}{1-p_{c}}\right)^{-1} \quad \chi>p_{c}
$$

The ratio $R / L$ can be considered as a tortuosity factor. The tortuosity factor reduces the actual pressure gradient with respect to the pressure gradient measured between two points in the porous medium and causes the fluids to make a detour. Hence its square enters in the permeability calculations. For the two-phase case a first power of the tortuosity may be preferred. The tortuosity factor $R / L$ tends to zero near the percolation threshold which can be seen from equations 2 and 3 .

We assume that the infinite clusters behave as parallel chains and disregard the permeability of chains perpendicular to the external pressure gradient. Therefore the permeability of each chain is determined as a conductivity of conductors in series i.e. the harmonic average permeability. The number of chains per unit crosssection of the porous medium $n(\chi)=R(\chi)^{-2}$.
Hence the effective permeability of the medium is the number of chains per unit cross-section times the tortuosity factor squared times the harmonic average i.e.

$$
\begin{aligned}
& k(\chi)=\frac{\int_{0}^{\infty} \psi(k) d k}{\int_{0}^{\infty} \frac{\psi(k)}{k} d k} \frac{R^{2}(x)}{L^{2}(x)} \frac{1}{R^{2}(x)}= \\
& \frac{\int_{0}^{\infty} \psi(k) d k}{L^{2}(\chi) \int_{0}^{\infty} \frac{\psi(k)}{k} d k}
\end{aligned}
$$

For a log-normally distributed permeability the above expression reduces to

$$
k(\chi)=\exp \left(\mu-\frac{1}{2} s^{2}\right)\left(\frac{\chi-p_{c}}{1-p_{c}}\right)^{2}
$$

where $\mu$ and $s^{2}$ are the average and variance of the log (permeability) field. For $\chi$ far away from the threshold value a geometric average is preferred. In this case the prefactor in equation 4 is $\exp (\mu)$. If, for other reasons, an arithmetic average is used the prefactor is $\exp \left(\mu+\frac{1}{2} s^{2}\right)$ (see also Desbarats (1990)).

\section{Viscous dominated case of two-phase displace- ment}

We will now give an extension for two-phase flow in the viscous dominated case. The applicability of the theory is restricted to viscous forces dominated flow of (averaged) thin reservoir sections. The capillary dominated case is elaborately studied by Yortsos (1991b, 1993). We will, however, state our result for the capillary dominated case in Appendix A because we use more simplifying assumptions. For the ease of understanding we will disregard the presence of shales.

When water enters the porous medium it will do so in the sequence of decreasing permeability. The porosity $\phi(k)$ can be a function of the permeability. Regions with a certain permeability $k$ will be either filled by water at the residual oil saturation $1-S^{\circ}(k)$ or by oil at the connate water saturation $S_{i}(k)$. In summary we use the following assumptions 
- Patterns are filled in order of decreasing permeability.

- Locally the displacement is piston like i.e. the pattern contains water, at residual oil saturation, or oil at connate water saturation. For these saturations the values of the local relative permeabilities equal the end point relative permeabilities or zero.

- The conductivity of the thus formed patterns can be used as the permeability in Darcy's law.

Let water have displaced oil from patterns with a permeability larger than $k_{s}\left(S_{w}\right)$. This statement defines $k_{s}$ and $p_{s}\left(k_{s}\left(S_{w}\right)\right)$ denotes the probability that we encounter a value of $k_{s}$ or higher (see Figure 2) i.e. $p_{s}=\int_{k_{s}}^{\infty} \psi(k) d k$.

Water only enters regions that form infinite clusters. For the density $(W)$ of infinite clusters (here containing water) we use a formula that is based on approximate results of Monte Carlo simulations on large grids and speculations of critical phenomena theory [Staufer (1985), Efros (1982), and Neimark (1986)]. We use that the density of infinite clusters obeys the power law near to the critical point $W \propto\left(p-p_{c}\right)^{\beta}$. Far away from the critical point i.e. for $p_{s}>>p_{c}$ the density of finite clusters $\left(p_{s}-W\right)$ (i.e. patterns with $k>k_{s}$ containing oil) tends to zero. The density of infinite clusters must be an increasing function of $p_{s}$. Clearly the density of infinite clusters must be zero for $p_{s} \leq p_{c}$. A function which satisfies all these requirements is

$$
\begin{array}{rc}
W\left(p_{s}\right)=p_{s c}\left(\frac{p_{s}-p_{c}}{p_{s c}-p_{c}}\right)^{\beta} & p_{c}<p_{s}<p_{s c} \\
W\left(p_{s}\right)=p_{s} & p_{s c}<p_{s}<1 \\
W\left(p_{s}\right)=0 & p_{s}<p_{c}
\end{array}
$$

where we use $p_{s c}=1.65 p_{c}$, which is the largest value that still satisfies the requirement that $W \leq p_{s}$. There is no water (on top of the connate water) in finite clusters because the water invades the porous medium through the infinite clusters.

A fraction (1-W) is occupied by oil. But the movable oil belongs again to infinite clusters. The density of infinite clusters for oil

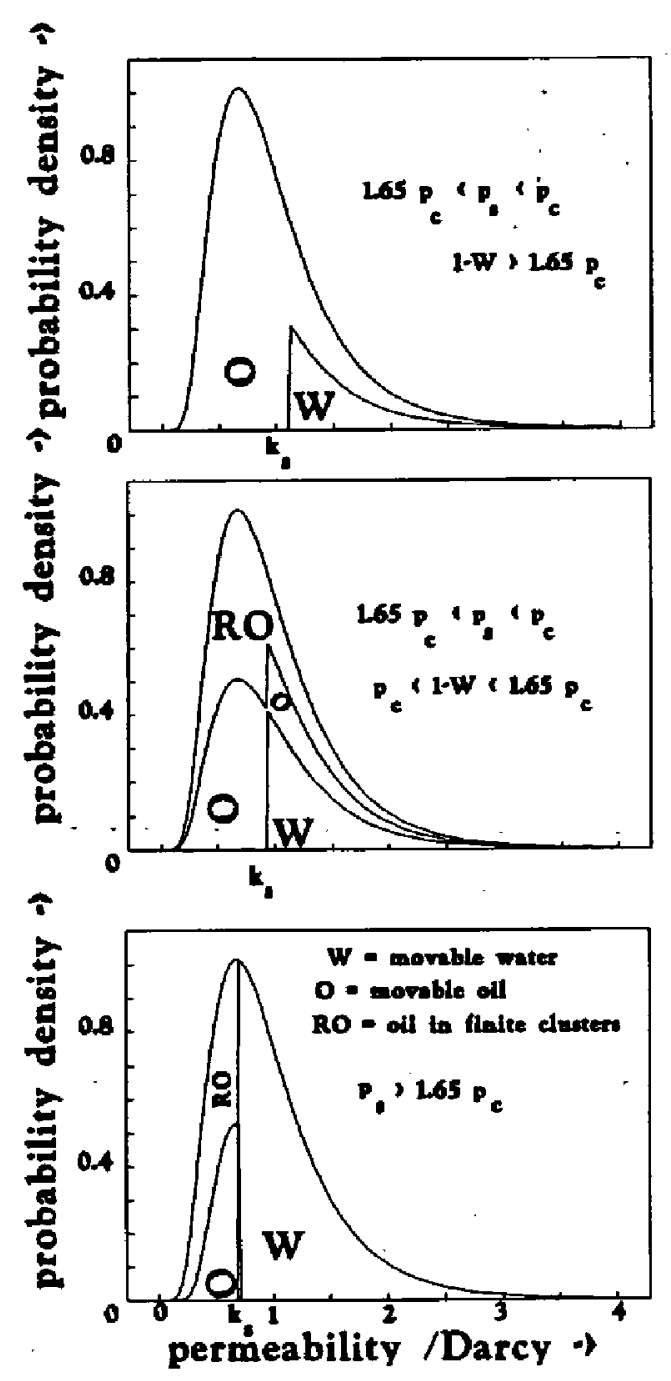

Figure 2: Parts of the distribution function $\psi(k)$ filled with water $W$, movable oil $O$, and oil in finite clusters $R O$ for values of $k_{s}$ dexreasing from the top to the bottom picture (see text).

$\left(O\left(W\left(p_{s}\right)\right)\right)$ has to satisfy the analogous conditions as $W\left(p_{s}\right)$ and we replace $p_{s}$ in equation 5 by $(1-W)$ i.e. $\left(O\left(W\left(p_{s}\right)\right)\right)=W(1-W)$. The distribution functions shown in figure 2 will be used to calculate the average water saturation and the average phase permeabilities within the infinite cluster below.

\subsection{Average water saturation}

Following the classical model of the waterflood in the stratified reservoir in the viscous dominated case [Hearn (1971)] we assume that the saturation in the water filled patterns behind the displacement front is $s^{\circ}(k)$, and the saturation in the non swept area upstream of the displacement front is $s_{i}(k)$. Of 
the areas with permeabilities higher than $k_{s}$ a fraction $\frac{W}{p_{s}}$ is filled with water and the rest $\left(\left(p_{s}-W\right) / p_{s}\right)$ is filled by oil. Hence the average water saturation $(E[S])$ is given in the equation

$$
\begin{array}{r}
\mathrm{E}[\phi] \mathrm{E}[\mathrm{S}]=\int_{0}^{k_{a}} s_{\mathrm{i}}(\mathrm{k}) \phi(\mathrm{k}) \psi(\mathrm{k}) \mathrm{dk} \\
+\int_{k_{s}}^{\infty} s^{o}(k) \phi(k) \frac{W}{p_{s}} \psi(k) d k \\
+\int_{k_{s}}^{\infty} s_{i}(k) \phi(k) \frac{p_{s}-W}{p_{s}} \psi(k) d k
\end{array}
$$

Here $E[\phi]=\int_{0}^{\infty} \phi(k) \psi(k) d k$ is the average porosity and $p_{s}=\int_{k_{a}}^{\infty} \psi(k) d k$.

\subsection{Pseudo relative phase per- meability}

For the calculation of the phase permeabilities we need to normalise the distribution functions for water $\psi_{w}(k)$ and for oil $\psi_{o}(k)$ (see also Figure 2).

As no water is present in patterns for which $k<k_{s}$ it follows that $\psi_{v}\left(k<k_{s}\right)=0$. Hence we use the cumulative probability $p_{s}$ to normalize the distribution function $\psi_{w}$.

$$
\begin{array}{r}
\psi_{w}(k)=0 \quad 0<k<k_{s} \\
\psi_{w}(k)=\frac{\psi(k)}{p_{s}} \quad k \geq k_{s}
\end{array}
$$

Similarly for the normalised distribution function for oil. Cumulatively a fraction (1-W) of all patterns with any permeability is filled with oil hence we need to normalise by dividing by $(1-W)$. Hence we obtain for $\psi_{o}(k)$

$$
\begin{aligned}
\psi_{o}(k)=\frac{\psi(k)}{1-W} & 0<k<k_{s} \\
\psi_{o}(k)=\psi(k) \frac{p_{s}-W}{p_{s}(1-W)} & k \geq k_{s}
\end{aligned}
$$

The permeability for each water filled site is $k k_{r w}(k)$. These sites are distributed with the density function given in equation 7 . In all other aspects the situation is completely analogous to the shale lenses (i.e. equation 4) and we obtain for the permeability of water

$$
k_{w}(E[S])=\frac{\int_{k,}^{\infty} \psi_{w}(k) d k}{L^{2}\left(\chi=p_{s}\right) \int_{k_{s}}^{\infty} \frac{\psi_{w}(k)}{k k_{r w}} d k}
$$

where we subsitute the fraction $p_{s}$ of patterns with permeability higher than $k_{s}$ instead of $\chi$ in expression 3 for the average length of the path between sites belonging to the same infinite cluster. We prefer to use $L\left(\chi=p_{s}\right)$ instead of $L(\chi=W)$. Indeed, W represents the fraction of the pattern filled by water in infinite clusters. That the water has not entered the finite clusters is a flow-technical aspect not dictated by percolation theory. The water conductivity (permeability) is also not affected whether the finite clusters are filled by water or oil. In the same way we obtain for the permeability of oil

$$
k_{\circ}(E[S])=\frac{\int_{0}^{\infty} \psi_{o}(k) d k}{L^{2}(\chi=1-W) \int_{0}^{\infty} \frac{\psi_{o}(k)}{k k_{\mathrm{ro}}} d k}
$$

where we have subsituted the fraction of oil filled patterns (1-W) oil filled clusters instead of $\chi$ in the expression 3 for the same reason as above. The integration in equations 9 and 10 must be performed numerically even for lognormal permeability distributions. Again we can also use a geometric average for $k_{\alpha}$

$$
k_{\alpha}(E[S])=\frac{\exp \left(\int_{0}^{\infty} \log \left(k k_{r \alpha}\right) \psi_{\alpha}(k) d k\right)}{L^{2}(\chi=\zeta)}
$$

where $\alpha=o, w$, and $\zeta=p$, if $\alpha=$ water and $\zeta=1-W$ if $\alpha=o i l)$. The arithmetic average

$$
k_{\alpha}(E[S])=\frac{\int_{0}^{\infty} k k_{r \alpha} \psi_{\alpha}(k) d k}{L^{2}(\chi=\zeta)}
$$

The formalism is easily adapted to describe the capillary dominated case (see appendix A).

\section{Comparison with layered models and capillary con- trolled models}

We want to compare to the stratified pseudo relative permeability model of Hearn (1971). 
Hearn considers a layered reservoir each with its own permeability. The layers are filled with the displacing fluid
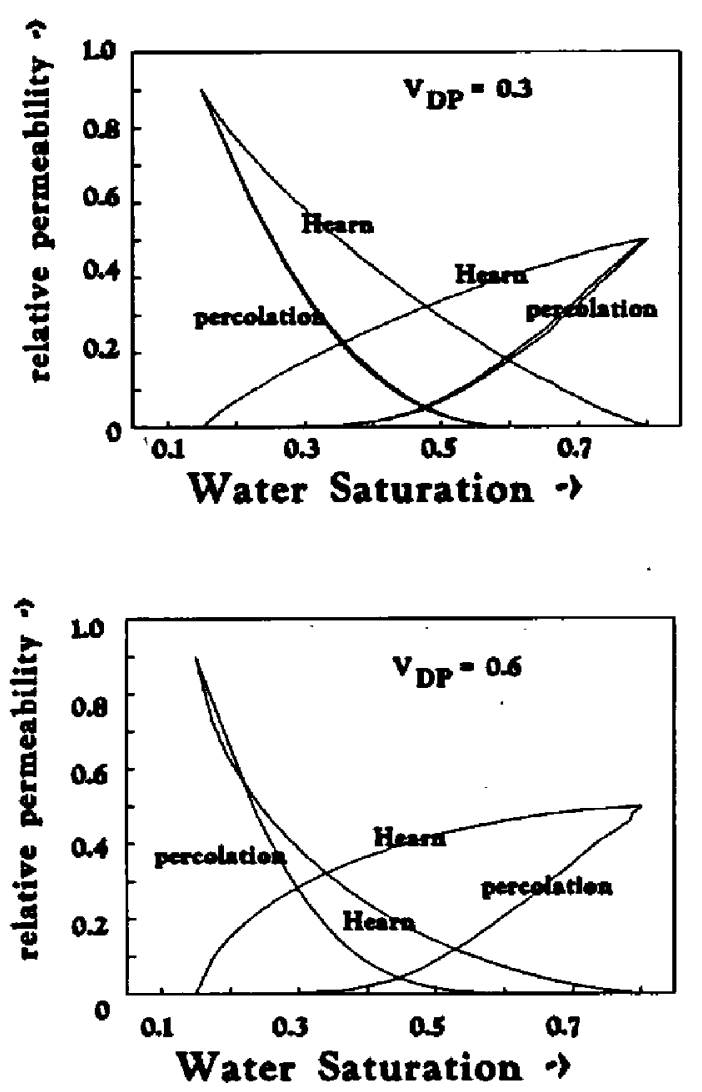

Figure 3: Relative arithmetically averaged (phase) permeabilities for the viscous dominated case; upper picture for $V_{D P}=0.3$. The difference between the harmonic- and the arithmetic average for the percolation case is hardly discernable; Lower picture for $V_{D P}=0.6$. Here the indicator Hearn refers to layers that are filled by the displacing fluid in the sequence of decreasing permeability.

from the high to the low permeable layers i.e. in order of decreasing permeability. In our notation we can summarise the model equations for the Hearn's model as follows

$$
\begin{aligned}
E[\phi] E[S] & =\int_{0}^{k_{s}} s_{i}(k) \phi(k) \psi(k) d k \\
& +\int_{k_{s}}^{\infty} s^{o}(k) \phi(k) \psi(k) d k(13)
\end{aligned}
$$

Again $E[\phi]=\int_{0}^{\infty} \phi(k) \dot{\psi(k)} d k$ is the average porosity. The (non-normalised) distribution function $\psi_{w h}(k)$ and $\psi_{o h}(k)$ are given by

$$
\psi_{w h}(k)=0 \quad 0<k<k_{s}
$$

$$
\begin{array}{rc}
\psi_{w h}(k)=\psi(k) & k \geq k_{s} \\
\psi_{o h}(k)=\psi(k) & 0<k<k_{s} \\
\psi_{o h}(k)=0 & k \geq k_{s}
\end{array}
$$

The phase permeability for water $\left(k_{\alpha=w}\right)$ and oil $\left(k_{\alpha=0}\right)$ is determined as a group of parallel conductors i.e. the arithmetic average

$$
k_{\alpha}(E[S])=\int_{0}^{\infty} k k_{r \alpha} \psi_{\alpha h}(k) d k
$$

The phase permeabilities calculated with equation 15 and with equation 12 are compared in Figure 3. We have made the comparison for two situations i.e. for a relatively homogeneous reservoir with $V_{D P}=0.3$ and a moderately heterogeneous reservoir with $V_{D P}$ $=0.6$. We have given the expressions for the capillary dominated case, i.e. when the layers are filled in the sequence of increasing permeability, in appendix A. A comparison with the layered model and the percolation model in the capillary controlled case is shown in Figure 4.

We also calculate the fractional flow function without the gravity term i.e.

$$
f(E[S])=\frac{1}{1+\frac{k_{o} \mu_{w}}{k_{w} \mu_{o}}}
$$

The fractional flow curves are compared for the viscous dominated case and the capillary controlled case all for a moderately heterogeneous $\left(V_{D P}=0.6\right)$ reservoir in Figure 5 .

\section{Discussion}

Geometric and harmonic averaging leads to a (non-physical) effective zero single phase permeability. With percolation theory a procedure is given to incorporate non-permeable parts. With our simplifications the procedure is extremely simple. The harmonic (or geometric average) of the permeable part (volume fraction $\chi$ ) needs to be multiplied by a reduction factor $L^{2}$ with $L=\frac{x-p_{c}}{1-p_{c}}$. The conductivity exponent of two in $\left(\left(\frac{x-p_{c}}{1-p_{c}}\right)^{2}\right)$ in equation 4 etc. is in agreement within the range of choices offered in the literature (see 

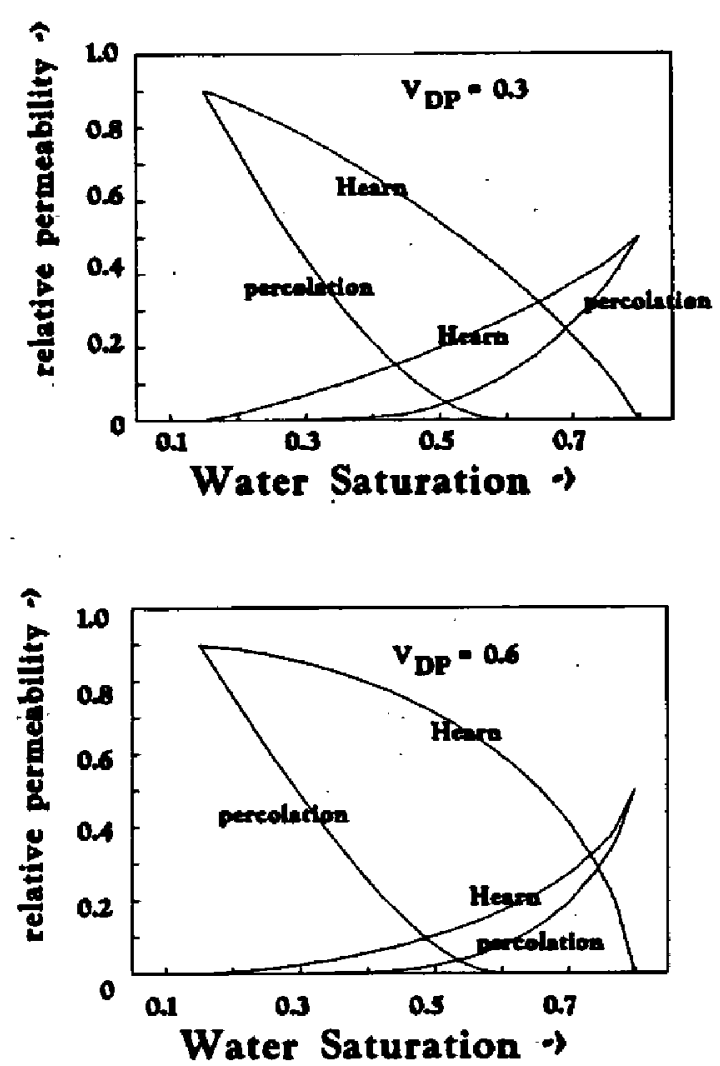

Figure 4: Relative arithmetically averaged (phase) permeabilities for the capillary dominated case; upper picture for $V_{D P}=0.3$. Lower picture for $V_{D P}=0.6$. Here, the indicator Hearn, refers to layers that are filled by the displacing fluid in the sequence of increasing permeability.

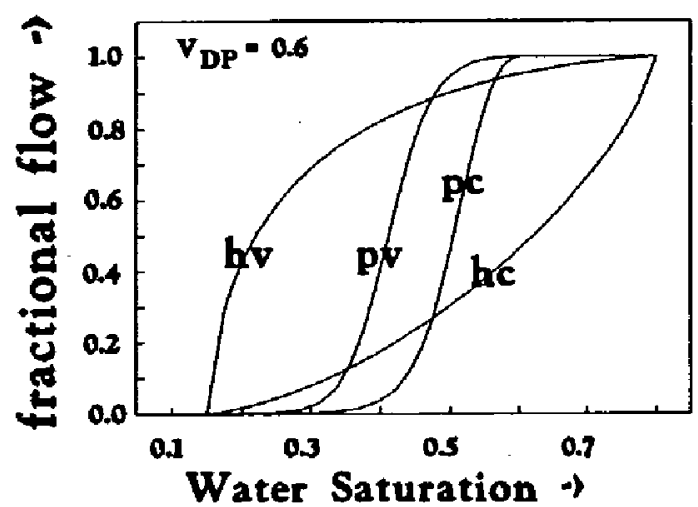

Figure 5: Fractional flow curves for the four cases considered i.e. $h v$ conventional Hearn model, $h c$ capillary dominated Hearn model, $p v$ viscous percolation model and $p c$ capillary percolation model $\left(V_{D P}=0.6\right)$. The mobility ratio $\frac{\mu_{0}}{\mu_{w}} \frac{k_{\text {ru }}}{k_{\text {ro }}}=1.67$
Staufer (1985) p52 \& p89). We note that if a tortuosity factor to the first power is used (which may be preferrable for the two phase case discussed below) the conductivity exponent becomes 1.9 instead of 2 i.e. leads only two a minor difference. A comparison to simulation results is required for fine tuning and validation. Whether the theory can be extended to describe the vertical to horizontal permeability ratio of reservoirs containing shale lenses is not yet clear (see Haldorson and Lake (1984)).

The two-phase (relative permeability) models discussed here have in common that they consider permeability patterns which are either filled up with the displacing fluid or with the displaced fluid. They differ only as to the sequence in which the patterns are filled up. Consequently the end-point permeabilities are the only aspect of the "microscopic" relative permeabilities that is retained. The model bears much similarity to the model of layered reservoirs proposed by Hearn (1971). Hearn considers the correlated case whereas percolation theory handles the completely uncorrelated case. In the correlated case the patterns are precisely filled up in the sequence of decreasing permeability (viscous dominated) or increasing permeability (capillary dominated). In the uncorrelated case certain high (low) permeable parts are isolated as they are surrounded by low (high) permeable parts and are thus not invaded by the displacing fluid. We have used percolation theory to deal with this aspect.

Figure 3 summarises the results for the viscous dominated case. Our example heterogeneity fields specified by the Dykstra and Parsons' coefficient $V_{D P}$ are less heterogeneous than the full field because the averaging (upscaling) comprises only small parts of the field. The water permeability for the layered (Hearn) model shows the characteristic concave shape. All other phase permeabilities have a convex shape. The percolation derived permeabilities are in general below the layer model derived permeabilities. This is due to the presence of isolated patterns in the percolation model that are not contributing to the permeability. For $V_{D P}=0.6$, 
however, the oil permeability of the percolation model creeps above the oil permeabilty due too a more favourable filling of highly permeable patterns by oil. The layered model shows typical oil wet behaviour but this is changed to indifferent wetting behaviour for the percolation model (both fluids appear to be wetting).

Figure 4 summarises the results for the capillary dominated case. Note that for the percolation model this does not boil down to an interchange of the oil with the water permeability and vice versa with Figure 3 (correcting for end point permeabilities and residual saturations) because all invading fluid is only present in non-isolated patterns. Now the oil permeability for the layered model has the concave shape. The layer model curves in the capillary dominated case are clearly representative for water wet conditions but this is reduced in the percolation model.

Figure 5 shows the fractional flow curves for a viscosity ratio $\frac{\mu_{0}}{\mu_{w}}=3$ and thus a mobility ratio $M=1.67$. From the fractional flow curves we can construct the saturation profile in 1$D$ flow. In general the saturation profile consists of a constant state part (a straight horizontal line), a rarefaction part (gradual decrease in water saturation), a shock part and a again constant state part. By inspection of Figure 5 (see Dake (1978), Welge (1952)) we observe that the saturation profile for the viscous dominated Hearn model case does not have a shock, whereas the capillary dominated Hearn model has no rarefaction part. For the viscous dominated percolation model there is both a rarefaction part and a shock. The capillary dominated percolation model has a somewhat smaller rarefaction part and a shock. A shock implies that part of the relperms cannot be reconstructed from the production and pressure history data [Johnson et al. (1959), Jones and Roszelle (1978)]. In general the percolation model shows a less favourable recovery for water flooding as oil remains trapped in isolated patches leading to an effective (porelevel + pattern level) residual oil saturation of $S_{\text {or,eff }}=0.45$. After establishment of local capillary equilibrium part of this oil trapped in patterns can still be produced. These aspects still need to be validated by a comparison to computer simulations and a comparison with the model of Kyte and Berry (1975).

\section{Conclusions}

1. Percolation theory provides a procedure to average (one-phase) permeability fields that contain a fraction of non-permeable parts.

2. Explicit formulae for the pseudo twophase permeabilities have been derived from the percolation model for the viscous dominated case.

3. The percolation model of two-phase flow bears conceptually much similarity to the layer model proposed by Hearn. The relative permeabilies obtained with the percolation model are lower than those obtained with Hearn's layer model. This can be attributed to the presence of isolated clusters not contributing to flow and the tortuosity factor.

4. The use of percolation theory for scale up of two-phase permeabilities is not restricted to the capillary dominated case.

5. The percolation model still requires fine tuning after validation with computer simulation studies and comparisons with other models.

\section{Nomenclature}

roman:

$E[] \quad$ expectation of []

$k$ permeability

$k_{\alpha} \quad$ phase (water or oil) permeability

$k_{r w} \quad$ endpoint rel. permeability for water (0.5)

$k_{\text {ro }} \quad$ endpoint rel. permeability for oil (0.8)

$L \quad$ length of cluster between neighbouring points

$p \quad$ cumulative probability of the bond percolation

$p_{c} \quad$ threshold probability

$k_{\mathrm{e}} \quad$ permeability corresponding to $p_{c}$

$k_{s} \quad$ minimum water filled permeability

$O \quad$ fraction of patterns with movable oil

$p_{c} \quad$ percolation threshold

$p_{s} \quad$ probability that $k>k_{s}$ 
$p_{s c} \quad p_{s c}=1.65 p_{c}$

$R$ distance between neighbouring occupied points

$S \quad$ water saturation

$s \quad$ standard deviation of $\log (\mathbf{k})$

$s_{i}(k)$ initial water saturation

$s^{o}(k) \quad$ water saturation in swept patterns

$W$ fraction of patterns with water

greek:

$\alpha \quad$ index meaning oil (o) or water (w)

$\beta \quad$ characteristic exponent $\beta=0.4$

$\phi \quad$ porosity

$\zeta \quad p_{s}$ for water and $(1-W)$ for oil

$\mu \quad$ Average of $\ln (k)$

$\nu \quad$ characteristic exponent $\nu=0.9$

$\psi \quad$ density distribution of permeability

$\chi \quad$ fraction occupied by permeable formation subscripts:

$h \quad$ according to theory of Hearn

o oil

$w \quad$ water

$\alpha \quad$ index meaning oil (o) or water (w)

\section{Acknowledgements}

We thank the Delft University of Technology for providing the funds to make the stay of Professor P. Bedrikovetsky possible.

\section{References}

1. Barenblatt, G.I, Entov, V.M., Ryzhik, V.M., 1990, Theory of Fluid Flows through Natural Rocks, Kluwer Academic Publishers, London \& Boston \& Dordrecht

2. Bedrikovetsky, P.G., 1993, Mathematical Theory of Oil and Gas Recovery (With applications to the ex-USSR oil and gas condensate fields), Kluwer Academic Publishers, London/Boston/Dordrecht

3. Bedrikovetsky, P.G. and Bruining, J., A Percolation Based Upscaling Technique for Relative Permeabilities and Capillary Pressure, TU-Report, Petroleum Engineering (1995).

4. Coats, K.H., Dempsey, J.R. and Henderson, J.H., 1971, The use of vertical equilibrium in two-dimensional reservoir performance, SPEJ, V.11, No.1, 63-71.
5. Desbarats, A.J., Spatial Averaging of Transmissivity, Proceedings of the Fifth Canadian/American Conference on Hydrology; Parameter Identification and Estimation for Aquifer and reservoir Characterization, Calgary, Alberta, Canada, Sept. 18-20 (1990).

6. Dietz, D.N., 1950, A theoretical approach to the problem of encroaching and bypassing edge water, Proc. Acad. Wetenschappen, Amsterdam, V56-B, 83.

7. Dykstra, H. and Parsons, R.L., The Prediction of Oil Recovery by Waterflood, API Secondary Recovery in the United States, API, Washington, DC (1950) 160-174.

8. Efros, A.L., 1982, Physics and Geometry of Chaos, Nauka, Moscow.

9. Haldorsen, H.H. and Lake, L.W., A New Approach to Shale Management in Field Scale Models, SPEJ Aug (1984) 447457.

10. Hearn, C.L., 1971, Simulation of stratified waterflooding by pseudo- relative permeability curves, JPT, 23, 805-814.

11. Heiba, A.A., Sahimi, M., Scriven, L.E. and Davis, H.T., Percolation theory of two-phase relative permeability, SPE paper No. 11015, presented at the 57th Annual Fall Technical Conference and Exhibition of the SPE of AIME in New Orleans, LA, Sept. 26- 28, 1982.

12. Heiba, A.A., Davis, H.T. and Scriven, L.E., Statistical network theory of threephase relative permeabilities, SPE paper No 12690, presented at the SPE/DOE Fourth Symposium on Enhanced Oil Recovery held in Tulsa, OK, April 1518, 1984.

13. Jensen, J.L. and Lake, L.W., The influence of Sample Size And Permeability Distribution on Heterogeneous Measures, SPERE (May 1988), 629-637.

14. Johnson, E.F., Bossler, D.C. and Naumann, V.O., 1959, Calculation of Relative Permeability from Displacement Experiments, J.Petr. Tech. 11, 61-63. 
15. Jones, S.C. and Roszelle, W.O., 1978, Gräphical Techniques for Determining Relative Permeability from Displacement Experiments, J.Petr. Tech. 30, 807817.

16. Kadet, V.V. and Selyakov, V.I., 1987, Percolation model of two-phase flow in porous media, Fluid Dynamics, No.1 (Transl. from Russian, 88-95).

17. Kanevskaya, R.D., 1988, Asymptotic analyses of the effect of capillary forces on the $2 D$ transfer of two-phase systems in porous media, Fluid Dynamics, No. 4 (Transl. from Russian, Izv. AN SSSR, Mekh. Jidk. i Gaza, 88-95).

18. Kyte, J.R. and Berry, D.W., New Pseudofunctions To Control Numerical Dispersion, SPEJ Aug (1975) 369-376.

19. Neimark, A.V., 1986, Percolation method of calculation of pore size distribution in meso porous materials based on the isotherms of adsorption and desorption, Physical Chemistry (USSR Academy of Sciences), V. LX, No 7, p.1745-1758.

20. Ryzhik, V.M., 1960, On capillary imbibition by water of an oil-saturated water-wet reservoir (in Russian), Izv.AN SSSR, OTN, Mekh. i Mash., N0 2, 149151

21. Selyakov, V.I. and Kadet, V.V., 1995, Percolation Models of Transport in Micro Heterogeneous Porous Media. With applications to geotechnology, Nedra, Moscow (in Russian)

22. Selyakov, V.I., 1986, Permeability of granulate and cavernous media, (in Russian), Phizika Zemli, No.12, 44-52.

23. Stauffer, D. and Aharony, A., 1992, Introduction to Percolation Theory, Taylor \& Francis, London, Washington, DC.

24. Welge, H.J., 1952, A Simplified Method for Computing Oil Recovery by Gas or Water Drive, Trans. AIME 195, 91-98.

25. Yortsos, Y.C., 1991a, A theoretical analysis of vertical flow equilibrium, SPE
Paper 22612, presented at the 66th SPE Annual Fall Meeting, Dallas, Texas (Oct. 6-9).

26. Yortsos, Y.C., Satic, C., Salin, D. and Bacri, J.-C. 1991b, Large Scale Averaging of Drainage at Local Capillary Control, SPE Paper 22592, presented at the 66th SPE Annual Fall Meeting, Dallas, Texas (Oct. 6-9).

27. Yortsos, Y.C., 1992, Analytical studies for processes at vertical equilibrium, 3rd European Conference on the Mathematics of Oil Recovery, Delft University of Technology, Delft, The Netherlands, June 17-19.

28. Yortsos, Y.C., Satic, C., Bacri, J.-C. and Salin, D., 1993, Transport in Porous Media, Vol. 10, No. 2, February, 171195

\section{A Equations for the cap- illary dominated case}

Here we give the equations for two-phase flow for the capillary dominated case (see also Yortsos $(1991 \mathrm{~b}, 1993))$. We use the same symbols as for the viscous dominated case but they have a slightly different meaning. We use the same assumptions as above but now Patterns are filled in order of increasing permeability. Let water have displaced oil from patterns with a permeability less than $k_{s}\left(S_{w}\right)$. 1 $p_{s}\left(k_{s}\left(S_{w}\right)\right)$ denotes the probability that we encounter a value of $k_{s}$ or less i.e. $p_{s}=$ $\int_{k_{s}}^{\infty} \psi(k) d k$. Water is only displaced from regions that form infinite clusters. In the same way as above we now have

$$
\begin{array}{rc}
W\left(p_{s}\right)=p_{s c}\left(\frac{1-p_{s}-p_{c}}{p_{s c}-p_{c}}\right)^{\beta} & p_{c}<1-p_{s}<p_{s c} \\
W\left(p_{s}\right)=1-p_{s} & p_{s c}<1-p_{s}<1 \\
W\left(p_{s}\right)=0 & 1-p_{s}<p_{c}(\mathrm{~A}-1)
\end{array}
$$

A fraction (1-W) is occupied by oil. For the calculation of the phase permeabilities we again need the normalised distribution functions $\psi_{w}(k)$ and $\psi_{o}(k)$.

As no water is present in patterns for which 
$k>k_{s}$ it follows that $\psi_{w}\left(k>k_{s}\right)=0$. Hence we use the cumulative probability $1-p_{s}$ to normalize the distribution function $\psi_{w}$.

$$
\begin{array}{cc}
\psi_{w}(k)=\frac{\psi_{w}(k)}{1-p_{s}} & 0<k<k_{s} \\
\psi_{w}(k)=0 \quad k \geq k_{s}
\end{array}
$$

The distribution function for oil $\psi_{o}(k)$ is now given by

$$
\begin{gathered}
\psi_{o}(k)=\frac{\psi(k)}{1-W} \quad k \geq k_{s} \\
\psi_{o}(k)=\psi(k) \frac{\left(1-p_{s}-W\right)}{\left(1-p_{s}\right)(1-W)} \\
0<k<k_{s}
\end{gathered}
$$

The average water saturation is now

$$
\begin{gathered}
E[\phi] E[S]=\int_{k_{o}}^{\infty} s_{i}(k) \phi(k) \psi(k) d k \\
+\int_{0}^{k_{s}} s_{i}(k) \phi(k) \frac{1-p_{s}-W}{1-p_{s}} \psi(k) d k \\
+\int_{0}^{k_{s}} s^{o}(k) \phi(k) \frac{W}{1-p_{s}} \psi(k) d k
\end{gathered}
$$

Here again $p_{s}=\int_{k_{s}}^{\infty} \psi(k) d k$. The phase permeability of water

$$
k_{w}(E[S])=\frac{\int_{0}^{k_{s}} \psi_{w}(k) d k}{L^{2}\left(\chi=1-p_{s}\right) \int_{0}^{k_{s}} \frac{\psi_{w}(k)}{k k_{r w}} d k}(\mathrm{~A}-5)
$$

In the same way we obtain for the phase permeability of oil again the expression 10

$$
k_{o}(E[S])=\frac{\int_{0}^{\infty} \psi_{o}(k) d k}{L^{2}(\chi=1-W) \int_{0}^{\infty} \frac{\psi_{o}(k)}{k k_{r o}} d k}(\mathrm{~A}-6)
$$

The equivalent expressions for the geometric and harmonic average are the same as equations 11 and 12 except that we use $L(\chi=$ $\left.1-p_{s}\right)$ for the water permeability.

The model equations for the stratified model are

$$
\begin{aligned}
E[\phi] E[S] & =\int_{k_{s}}^{\infty} s_{i}(k) \phi(k) \psi(k) d k \\
& +\int_{0}^{k_{o}} s^{o}(k) \phi(k) \psi(k) d k
\end{aligned}
$$

The (non-normalised) distribution function $\psi_{w h}(k)$ and $\psi_{o h}(k)$ are given by

$$
\begin{array}{cc}
\psi_{w h}(k)=\psi(k) & 0<k<k_{s} \\
\psi_{w h}(k)=0 & k \geq k_{s} \\
\psi_{o h}(k)=0 & 0<k<k_{s} \\
\psi_{o h}(k)=\psi(k) & k \geq k_{s}
\end{array}
$$

The phase permeabilities for water $\left(k_{\alpha=w}\right)$ and oil $\left(k_{\alpha=0}\right)$ are determined as a group of parallel conductors, i.e. the arithmetic average.

$$
k_{\alpha}(E[S])=\int_{0}^{\infty} k k_{r \alpha} \psi_{\alpha h}(k) d k
$$

